\title{
Healthcare-associated infection prevention and control management in a tertiary hospital and an overall evaluation
}

\author{
Weiping Liu, Tianhui Guo, Haoxue Li, Yuping Zhao, Kai Zhang, Yunting Hai, Yueying Jiao, \\ Huimin Xing, Binbin Xu, Haibo Bai, Huan Bao, Shuai Zhang, Wei Ren, Lifang Yang, Huijun Yang, \\ Junwei Tian, Meng Wang, Yongfang Yang \\ Department of Nosocomial Infection Control, Inner Mongolia People’s Hospital, Hohhot, China \\ Contributions: (I) Conception and design: W Liu, T Guo; (II) Administrative support: W Liu, Y Jiao, H Xing; (III) Provision of study materials or \\ patients: K Zhang, Y Hai, J Tian, H Yang; (IV) Collection and assembly of data: B Xu, H Bai, S Zhang, H Bao, M Wang, L Yang; (V) Data analysis \\ and interpretation: Y Zhao, Y Yang, H Li, W Ren; (VI) Manuscript writing: All authors; (VII) Final approval of manuscript: All authors. \\ Correspondence to: Yongfang Yang, BA. Department of Nosocomial Infection Control, Inner Mongolia People's Hospital, No. 20 of Zhaowuda Street, \\ Saihan District, Hohhot 010017, China. Email: yyf1162265076@163.com.
}

Background: Healthcare-associated infection (HAI) is a crucial factor influencing medical quality. Studies about HAI management situations are rare, especially for the Inner Mongolia region of China. Therefore, this study aimed to investigate management procedures and the overall evaluation of HAI in order to inform HAI management improvement more scientifically.

Methods: A questionnaire was used to investigate HAI-related prevention and control indicators in tertiary hospitals in the Inner Mongolia region from July 2018 to June 2019.

Results: The survey showed that the mean incidence rate of HAI was $3.79 \%$. The mean rate of hand hygiene compliance of healthcare workers (HCWs), inpatient's antibiotics-use rate, and the detection of the antibiotic ratio before therapy was $54.34 \%, 34.33 \%$, and $25.40 \%$, respectively. The mean of the surgical site infection (SSI) rate of the level I incision and the preventive antibiotics-use ratio of the level I incision was $1.31 \%$ and $28.89 \%$, respectively. The mean of the multi-drug resistant organism (MDRO) infection rate was $0.40 \%$ and the mean of the MDRO detection rate was $18.55 \%$. The mean of the central line-associated bloodstream infection rate was $2.24 \%$, the ventilator-associated pneumonia (VAP) rate was $11.17 \%$, and the catheter-associated urinary tract infection (CAUTI) rate was $1.95 \%$. As for the overall evaluation, 19 (35.85\%) hospitals had a bad grade, 18 (33.96\%) hospitals had a medium grade, and $16(30.19 \%)$ hospitals had a good grade.

Conclusions: The incidence rate of HAI in tertiary hospitals in the Inner Mongolia region is higher than the national level. Also, the overall evaluation of bad-grade hospitals and their deficiencies should be used as an example to improve the HAI management level.

Keywords: Healthcare-associated infection management (HAI management); healthcare-associated infection quality control indicator (HAI quality control indicator); healthcare-associated infection prevention and control (HAI prevention and control); overall evaluation

Submitted Jan 06, 2020. Accepted for publication Jun 03, 2020.

doi: 10.21037/apm-20-65

View this article at: http://dx.doi.org/10.21037/apm-20-65 


\section{Introduction}

Healthcare-associated infection (HAI) is a threat to inpatient's medical safety and can negatively affect the medical quality of hospitals. More attention has been paid to HAI management, and an independent HAI management department has been established in most healthcare facilities in order to control HAI. Since HAI management in China began later than in developed countries, several problems, including management instability, surveillance incompleteness, and healthcare workers' (HCWs) low awareness of HAI, remain unresolved (1-6). Inner Mongolia is a considered a developing region in northern China. Understanding the current status and identifying the weak links in HAI management within Inner Mongolia could improve HAI management and decrease the incidence rate of HAI, shorten the average length of stay (LOS), improve the circulation speed of medical facilities, reduce the economic burden of patients, and promote the hospital's social and economic influence. Despite this need, little research concerning HAI management is available, especially related to the Inner Mongolia region (7-19). In order to understand the HAI management situation, we conducted a survey of 11 indicators as outlined by the National Health Commission's HAI management requirements, considered to comprehensively reflect the HAI management situation. This survey included 53 tertiary hospitals in 12 cities, covering all cities in Inner Mongolia. Rank-sum ratio (RSR) was used to evaluate HAI management situation overall.

\section{Methods}

\section{Sampling method}

The sample included all the tertiary hospitals in the Inner Mongolia Autonomous Region, with a total of 53 hospitals in 12 cities. Each city had at least 1 tertiary hospital. The 53 hospitals covered all areas in the Inner Mongolia Autonomous Region, which is in northern China. The study was approved by the Ethics Board of Inner Mongolia People's Hospital (No. 202000303L).

\section{Survey}

According to HAI the management quality control indicator section in the National Health Commission's notification of quality control indicator of anesthesiology and other six majors (20), 11 indicators were selected as an evaluation of HAI prevention and control effect including the incidence rate of HAI, multi-drug resistant organism (MDRO) infection rate, MDRO detection rate, hand hygiene compliance of $\mathrm{HCW}$, inpatients' antibiotics-use rate, antibiotics detection ratio before therapy, surgical site infection (SSI) rate of the level I incision, the preventive antibiotics-use ratio of the level I incision, central lineassociated bloodstream infection rate, ventilator-associated pneumonia (VAP) rate, and catheter-associated urinary tract infection (CAUTI) rate.

\section{Definitions}

HAI was defined as an infection present at the time of hospital admission or within 48 hours of admission.

Indicators to assess the HAI situation were defined according the formulas given below:

* Incidence rate of HAI = existing HAI case number/ total inpatient number in the same period $\times 100 \%$;

- MDRO infection rate $=$ MDRO infection case number/ total inpatient number in the same period $\times 100 \%$;

- MDRO detection rate = strain number of MDRO detection number/strain number of the same organism detection number $\times 100 \%$;

* Hand hygiene compliance of $\mathrm{HCW}=$ number of times hand hygiene was performed/number of times hand hygiene was investigated $\times 100 \%$;

- Inpatients' antibiotics-use rate $=$ number of antibiotics used by an inpatient/total inpatient number in the same period $\times 100 \%$;

* Antibiotics detection ratio before the therapy = case number of antibiotics detected before therapy/total number of antibiotics used by inpatients in the same period $\times 100 \%$;

* SSI rate of the level I incision = case number of SSI rate of the level I incision/total patient number of the patient level I incision $\times 100 \%$;

* The preventive antibiotics-use ratio of the level I incision = patient number of the preventive antibiotics-used ratio of the level I incision/total patient number of the patient level I incision $\times 100 \%$;

* Central line-associated bloodstream infection $($ CLABSI $)$ rate $=$ case number of CLABSI/total days that the central line was used $\times 100 \%$;

* VAP rate $=$ case number of $\mathrm{VAP} /$ total days that the ventilator was used $\times 100 \%$;

* CAUTI rate $=$ case number of CAUTI/total days that the catheter was used $\times 100 \%$. 


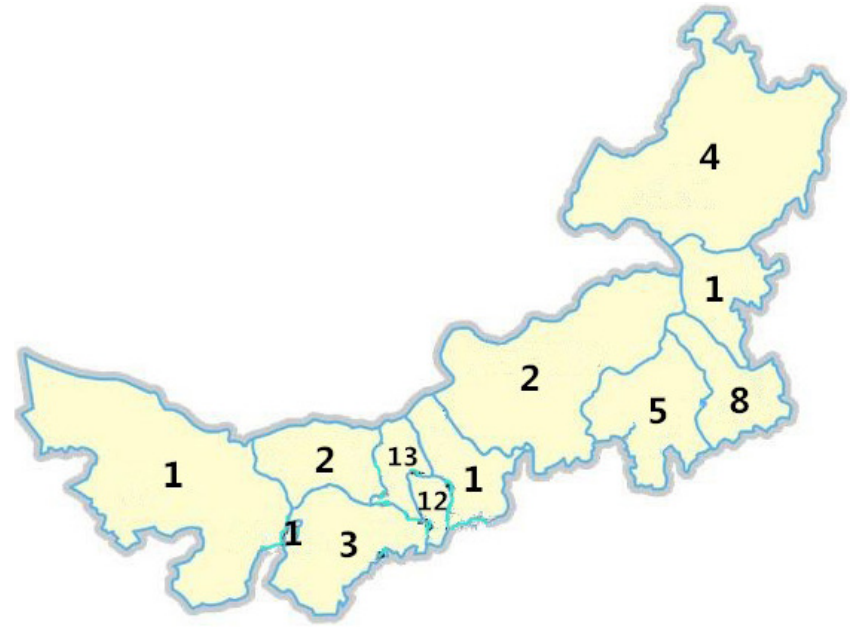

Figure 1 Distribution of hospitals surveyed.

Table 1 Characteristic of hospitals surveyed

\begin{tabular}{lcc}
\hline Hospital classification & Number & $\%$ \\
\hline Hospital level & 6 & 11.32 \\
Provincial hospital & 47 & 88.68 \\
Municipal hospital & & \\
Teaching hospital & 42 & 79.25 \\
Yes & 11 & 20.75 \\
No & & \\
Hospital type & 34 & 64.15 \\
General hospital & 10 & 18.87 \\
Specialized hospital & 9 & 16.98 \\
Tradition Chinese or traditional Mongolian & 53 & 100.00 \\
Total & & \\
\hline
\end{tabular}

\section{RSR calculation}

Probability unit Probit was used to express the distribution of RSR, which is the total downward accumulative frequency of the given RSR values. The protocol for the calculation of Probit is as follows: (I) design a RSR frequency distribution table, list the frequency $(f)$ of different groups, and calculate the accumulative total frequency $(\Sigma f)$ of a given group; (II) calculate the rank $(R)$ and average rank $(A R)$ of the RSR of a given group; (III) calculate the downward accumulative total frequency $(p=\mathrm{AR} / \mathrm{n})$, and switch the percentage $(p)$ to the frequency unit Probit. Probit should correspond with the standard normal deviation $(u)$ of the percentage $(p)$ plus 5 .

\section{Data organization and analysis}

EpiData3.0 was used to build the database, Microsoft Excel 2010 was used to organize the data, SPSS 19.0 was used for the data analysis, and a $\mathrm{P}$ value $<0.05$ was considered as statistically significant.

Non-parametric RSR was used to conduct an overall evaluation of HAI prevention and control quality, and RSR was used to summarize the overall level of several indicators.

\section{Results}

\section{Distribution and characteristic of hospitals}

A total of 53 hospitals, covering all cities in the Inner Mongolia Autonomous Region, were included in the analysis, with the distributional number being shown in Figure 1. Most of the hospitals in this study were municipal hospitals $(88.68 \%)$, teaching hospitals $(79.25 \%)$, and general hospitals (54.15\%) (Table 1).

\section{HAI management quality control indicator}

The survey showed that the highest, lowest, and average incidence rate of HAI was $5.03 \%, 3.02 \%$, and $3.79 \%$, respectively. The mean of hand hygiene compliance of HCW, inpatients' antibiotics-use rate, and antibiotics detection ratio before therapy were $56.34 \%, 34.33 \%$, and $25.40 \%$, respectively. The mean SSI rate of the level I incision was $1.31 \%$, and the preventive antibiotics-use ratio of the level I incision was $28.89 \%$. The mean of MDRO infection rate was $0.40 \%$, and the mean of MDRO detection rate was $18.55 \%$. The mean rate of the central line-associated bloodstream infection was $2.24 \%$, the VAP was $11.17 \%$, and the CAUTI was $1.95 \%$. These details are shown in Table 2.

\section{Overall evaluation of HAI prevention and control quality}

\section{Indicators and RSR}

Among the 11 indicators, hand hygiene compliance of HCW (Y4) and antibiotics detection ratio before therapy (Y6) were ranked from low to high. Other indicators including incidence rate of HAI (Y1), MDRO infection rate (Y2), MDRO detection rate (Y3), inpatients' antibiotics-use rate (Y5), SSI rate of the level I incision (Y7), the preventive 
Table 2 Indicator distribution

\begin{tabular}{|c|c|c|c|}
\hline Quality control indicator & Mean \pm SD & Maximum & Minimum \\
\hline MDRO infection rate & $0.004 \pm 0.001$ & 0.006 & 0.002 \\
\hline MDRO detection rate & $0.186 \pm 0.019$ & 0.220 & 0.149 \\
\hline Hand hygiene compliance of HCW & $0.563 \pm 0.053$ & 0.658 & 0.481 \\
\hline Antibiotics detect ratio before therapy & $0.254 \pm 0.030$ & 0.309 & 0.201 \\
\hline SSI rate of level incision & $0.013 \pm 0.002$ & 0.016 & 0.010 \\
\hline Preventive antibiotics use ratio of level I incision & $0.289 \pm 0.005$ & 0.299 & 0.280 \\
\hline Central line associated blood stream infection rate & $0.022 \pm 0.001$ & 0.025 & 0.020 \\
\hline
\end{tabular}

HAI, healthcare-associated infection; MDRO, multi-drug resistant organism; HCW, healthcare worker; SSI, surgical site infection; CAUTI, catheter-associated urinary tract infection.

antibiotics-use ratio of the level I incision (Y8), central lineassociated bloodstream infection rate (Y9), VAP rate (Y10), and CAUTI rate (Y11) were ranked from high to low. The average rank value was used for the same indicator of the same value.

Calculations included RSR by a matrix of 11 indicators and rank hospitals by RSR values (Table 3).

\section{Distribution of RSR}

The distribution of RSR (probability unit Probit), average rank $A R$, and downward accumulative total frequency $\mathrm{P}$ was calculated based on the abovementioned method. The RSR values were between $0.223-0.801$ and the Probit values were between 2.922-7.078. These details are shown in Table 4 .

\section{Regression equation}

To calculate the regression equation, the accumulative total frequency corresponding probability unit, Probit, was used as the independent variable while RSR value was used as the dependent variable. The linear regression equation was as follows: RSR $=\mathrm{A}+\mathrm{B} \times$ Probit. According to the previous results of RSR and Probit, the regression equation was calculated as follows: $\mathrm{RSR}=-0.67482+0.23532$ Probit $(\mathrm{F}=1425.68, \mathrm{P}<0.001)$.

\section{Grade and rank}

Grade and rank evaluations were given according to the RSR value. In addition, the three-grade method was used in this research; grade function and corresponding frequency unit cut-off values were based on medical biostatistics version 4 (Editor: Sun Zhenqiu). These details are shown in Table 5. After that, grade and rank evaluations were given according to the graded probability unit Probit value and the corresponding RSR estimated value based on the regression function calculation. The grade and rank results are shown in Table 5.

\section{Discussion}

The incidence rate of HAI was associated with scale, class, and the patient's situation in the hospital. The mean incidence rate of HAI from this survey was higher than both the national level (1.58\%) and the result of a survey conducted in 184 tertiary hospitals in 2016 (21) that also had a result higher than $2.73 \%$ in Guizhou province (22). Furthermore, hand hygiene compliance of HCW, antibiotics detection ratio before therapy, the SSI rate of the level I incision, the preventive antibiotics-use ratio of the level I incision, MDRO infection rate, MDRO detection rate, and the VAP rate were lower than the rates found in other regions (23-26). The inpatients' antibiotics-use rate and the CAUTI rate was the same as the national level. Lastly, the central line-associated bloodstream infection rate was higher than the national level (27-29).

The incidence rate is a direct reflection of HAI management quality. The principle aim of HAI management 
Table 3 Rank and the RSR of indicators

\begin{tabular}{|c|c|c|c|c|c|c|c|c|c|c|c|c|}
\hline $\begin{array}{l}\text { Hospital } \\
\text { number }\end{array}$ & $\mathrm{Y} 1$ & Y2 & Y3 & Y4 & Y5 & Y6 & Y7 & Y8 & Y9 & Y10 & Y11 & RSR \\
\hline 1 & 45 & 48 & 44 & 51 & 44 & 51 & 49 & 53 & 42 & 50 & 50 & 0.903 \\
\hline 3 & 33.5 & 17.5 & 25 & 52 & 37 & 32 & 19.5 & 51 & 29 & 44 & 34 & 0.642 \\
\hline 4 & 37 & 45.5 & 39 & 36 & 39 & 53 & 52 & 49 & 46 & 28 & 38.5 & 0.794 \\
\hline 6 & 31.5 & 52 & 41 & 41 & 45 & 50 & 50 & 48 & 39 & 48 & 50 & 0.850 \\
\hline 7 & 22 & 17.5 & 35 & 4 & 17 & 33 & 17 & 37 & 16 & 16.5 & 27 & 0.415 \\
\hline 8 & 25 & 37 & 5 & 42 & 25 & 47 & 23.5 & 21 & 29 & 13 & 19 & 0.491 \\
\hline 12 & 22 & 32 & 6 & 22 & 46 & 35 & 37.5 & 35.5 & 18.5 & 33.5 & 30 & 0.545 \\
\hline 13 & 4 & 2.5 & 1 & 2 & 2 & 2 & 1 & 1 & 4 & 3 & 13.5 & 0.062 \\
\hline 14 & 15 & 7 & 28 & 11 & 10 & 27 & 21.5 & 11.5 & 23.5 & 4 & 24 & 0.313 \\
\hline 15 & 17 & 5.5 & 23.5 & 21 & 38 & 26 & 25.5 & 34 & 14 & 15 & 27 & 0.423 \\
\hline 16 & 43.5 & 50 & 45 & 38 & 43 & 49 & 48 & 27 & 47.5 & 46.5 & 44 & 0.826 \\
\hline 17 & 16 & 40.5 & 15.5 & 39 & 31 & 29 & 31 & 33 & 44 & 8.5 & 24 & 0.534 \\
\hline 18 & 5 & 2.5 & 9.5 & 18 & 4 & 24 & 28.5 & 15.5 & 36.5 & 38.5 & 24 & 0.353 \\
\hline 24 & 18 & 11 & 36 & 28 & 19 & 18 & 27 & 51 & 29 & 31 & 16.5 & 0.488 \\
\hline 25 & 40 & 20.5 & 30 & 23 & 30 & 20 & 17 & 9 & 8.5 & 19 & 10 & 0.389 \\
\hline 26 & 31.5 & 52 & 48 & 34 & 50 & 42 & 47 & 39 & 50.5 & 45 & 47 & 0.834 \\
\hline 27 & 38.5 & 37 & 27 & 20 & 21.5 & 23 & 37.5 & 18 & 12 & 7 & 34 & 0.473 \\
\hline 28 & 3 & 10 & 2 & 3 & 8 & 4 & 4.5 & 5 & 2.5 & 1 & 5 & 0.082 \\
\hline 29 & 52 & 44 & 43 & 43 & 32 & 44 & 37.5 & 35.5 & 35 & 46.5 & 47 & 0.788 \\
\hline 30 & 13 & 2.5 & 19 & 24 & 20 & 19 & 17 & 14 & 21 & 42.5 & 2 & 0.333 \\
\hline 31 & 24 & 2.5 & 32 & 33 & 7 & 21 & 8 & 19.5 & 10 & 16.5 & 10 & 0.315 \\
\hline 32 & 11 & 20.5 & 14 & 50 & 6 & 22 & 25.5 & 10 & 16 & 10.5 & 6 & 0.328 \\
\hline 33 & 46 & 47 & 46 & 53 & 27 & 46 & 42 & 47 & 49 & 22 & 44 & 0.804 \\
\hline 34 & 30 & 15.5 & 8 & 9 & 21.5 & 16 & 12 & 17 & 34 & 6 & 34 & 0.348 \\
\hline
\end{tabular}

Table 3 (continued) 
Table 3 (continued)

\begin{tabular}{|c|c|c|c|c|c|c|c|c|c|c|c|c|}
\hline $\begin{array}{l}\text { Hospital } \\
\text { number }\end{array}$ & $\mathrm{Y} 1$ & Y2 & Y3 & Y4 & Y5 & Y6 & Y7 & Y8 & Y9 & Y10 & Y11 & RSR \\
\hline 35 & 47 & 37 & 50.5 & 35 & 48 & 43 & 44 & 23.5 & 53 & 40.5 & 41 & 0.793 \\
\hline 37 & 50 & 35 & 52 & 5 & 52 & 39 & 44 & 29.5 & 38 & 38.5 & 41 & 0.727 \\
\hline 40 & 2 & 13 & 20 & 13 & 12 & 9 & 14.5 & 25 & 5.5 & 40.5 & 13.5 & 0.288 \\
\hline 41 & 29 & 28.5 & 17 & 26 & 26 & 15 & 40.5 & 42.5 & 40.5 & 23 & 19 & 0.527 \\
\hline 42 & 1 & 23.5 & 11.5 & 6 & 3 & 8 & 2 & 2.5 & 12 & 32 & 16.5 & 0.202 \\
\hline 46 & 36 & 26.5 & 22 & 16 & 16 & 13 & 8 & 15.5 & 18.5 & 10.5 & 2 & 0.315 \\
\hline 47 & 22 & 13 & 7 & 7.5 & 5 & 12 & 10.5 & 2.5 & 12 & 27 & 7 & 0.215 \\
\hline 48 & 7 & 26.5 & 29 & 25 & 24 & 6 & 4.5 & 6 & 2.5 & 42.5 & 13.5 & 0.320 \\
\hline 49 & 14 & 33.5 & 34 & 19 & 18 & 10 & 40.5 & 41 & 5.5 & 35 & 21.5 & 0.467 \\
\hline 50 & 19 & 40.5 & 50.5 & 48 & 40 & 41 & 31 & 29.5 & 25.5 & 51 & 52.5 & 0.735 \\
\hline 51 & 26 & 23.5 & 9.5 & 15 & 23 & 11 & 14.5 & 11.5 & 50.5 & 8.5 & 4 & 0.338 \\
\hline 52 & 9 & 13 & 4 & 14 & 9 & 14 & 10.5 & 19.5 & 44 & 37 & 8 & 0.312 \\
\hline
\end{tabular}

RSR, rank-sum ratio.

Table 4 distribution of RSR, AR, P and Probit

\begin{tabular}{lccc}
\hline Item & Maximum & Median & Minimum \\
\hline RSR & 0.801 & 0.472 & 0.223 \\
AR & 53 & 27 & 1 \\
P & 1 & 0.509 & 0.019 \\
Probit & 7.078 & 5 & 2.922 \\
\hline
\end{tabular}

RSR, rank-sum ratio; $A R$, average rank.

Table 5 Cut-off value of grade and rank

\begin{tabular}{lcccc}
\hline Type & Px & Probit & RSR & $\begin{array}{c}\text { Hospital } \\
\text { number }\end{array}$ \\
\hline T1 (bad) & $<15.866$ & $<4$ & $<0.353$ & 19 \\
T2 (medium) & $15.866-84.134$ & $4-6$ & $0.353-0.642$ & 18 \\
T3 (good) & $>84.134$ & $>6$ & $>0.642$ & 16 \\
\hline
\end{tabular}

RSR, rank-sum ratio. is to reduce the incidence rate of HAI. Hand hygiene and rational antibiotic use are 2 crucial measures for preventing and controlling HAI $(13,30)$. MDRO infection, SSI, VAP, CAUTI, and central line-associated bloodstream infection are several main infection types of HAI (31-33). The effective control rate of these infections results in the effective control of the incidence rate of HAI $(8,34)$. Therefore, investigating 11 HAI management indicators could comprehensively reflect the HAI management situation, and evaluation based on these $11 \mathrm{HAI}$ management indicators could provide an overall assessment of HAI management quality of a given hospital.

Medical care evaluation is an important method and type of evidence in hospital quality control. An overall evaluation can be completed by quantifying multiple factors of medical care quality and ranking these evaluation objects to provide evidence of medical judgment (35). Research 
has shown that RSR is an overall evaluation method which has practical application, and it has been widely used in hospital quality evaluation (36). Using RSR to evaluate the overall evaluation has the advantage of not needing specific requirements of indicator and evaluation objects. RSR has no unit, and so rank calculation can eliminate interference of abnormal values (37). The present study is the first to use RSR for the overall evaluation off HAI management quality. Evaluating HAI management's using several quality indicators by RSR is simple, practicable, and objective. It is thus a scientifically sound method for effectively improving HAI management and can be used as an alternative evaluation tool for health administrations' assessment or selection.

\section{Conclusions}

This study included all tertiary hospitals in Inner Mongolia Autonomous Region, which has high quality medical care, in its survey. We chose 11 indicators with high importance, that we were confident could reflect HAI prevention and control quality; these indicators could comprehensively reflect HAI management quality overall, and its evaluation result has reference value. Our findings showed 19 (35.85\%) hospitals had a bad grade, 18 (33.96\%) hospitals had a medium grade, and 16 (30.19\%) hospitals had a good grade. Hospitals with bad grades should enhance their management and improve their HAI prevention and control measures. Based on a survey of 11 HAI management indicators, Rank Sum Ration was used to overall evaluating the HAI management status of each hospital in the study. Overall evaluation based on Rank Sum Ration is a scientific evaluation tool of hospital HAI management quality assessment.

Since the current status of HAI management in Inner Mongolia has been seldom discussed, we endeavored to provide a comprehensive survey that included $11 \mathrm{HAI}$ management indicators. From this we derived a greater understanding of the HAI management situation in Inner Mongolia. Based on this, the advantages and weaknesses of HAI management in Inner Mongolia could be further discussed in a future study which could provide scientific evidence to inform the improvement of HAI management in the region.

\section{Acknowledgments}

Funding: This work is supported by Department of Human
Resources and Social Security of Inner Mongolia; State Key Laboratory for Infectious Disease Prevention and Control (2019SKLID305); Department of Science \& Technology of Inner Mongolia [No. 2017MS(LH)0845]; Infection Prevention and Control Research Fund Administration Commission of China Geriatric Society (No. GRYJLRK2018021); and Health Commission of Inner Mongolia (No. 201703006).

\section{Footnote}

Data Sharing Statement: Available at http://dx.doi. org/10.21037/apm-20-65

Conflicts of Interest: All authors have completed the ICMJE uniform disclosure form (available at http://dx.doi. org/10.21037/apm-20-65). The authors have no conflicts of interest to declare.

Ethical Statement: The authors are accountable for all aspects of the work in ensuring that questions related to the accuracy or integrity of any part of the work are appropriately investigated and resolved. The study was approved by the Ethics Board of Inner Mongolia People's Hospital (No. 202000303L).

Open Access Statement: This is an Open Access article distributed in accordance with the Creative Commons Attribution-NonCommercial-NoDerivs 4.0 International License (CC BY-NC-ND 4.0), which permits the noncommercial replication and distribution of the article with the strict proviso that no changes or edits are made and the original work is properly cited (including links to both the formal publication through the relevant DOI and the license). See: https://creativecommons.org/licenses/by-nc-nd/4.0/.

\section{References}

1. Dramowski A, Cotton MF, Whitelaw A. Surveillance of healthcare-associated infection in hospitalised South African children: Which method performs best? S Afr Med J 2016;107:56-63.

2. Koulenti D, Tsigou E, Rello J. Nosocomial pneumonia in 27 ICUs in Europe: perspectives from the EU-VAP/CAP study. Eur J Clin Microbiol Infect Dis 2017;36:1999-2006.

3. Dudeck MA, Weiner LM, Allen-Bridson K, et al. National Healthcare Safety Network (NHSN) report, data summary for 2012, Device-associated module. Am J Infect Control 
2013;41:1148.

4. Loveday HP, Wilson JA, Pratt RJ, et al. epic3: national evidence-based guidelines for preventing healthcareassociated infections in NHS hospitals in England. J Hosp Infect 2014;86:S1-70.

5. Kullin B, Meggersee R, D'Alton J, et al. Prevalence of gastrointestinal pathogenic bacteria in patients with diarrhoea attending Groote Schuur Hospital, Cape Town, South Africa. S Afr Med J 2015;105:121-5.

6. European Centre for Disease Control. EUCLIDEuropean multicenter prospective bi-annual point prevalence study of Clostridium difficile infection in hopsitalised patients with diarrhoea: the fuller picture of the incidence of Clostridium difficile infection (CDI) across Europe. Solna: ECDC, 2014.

7. Haley RW, Culver DH, White JW, et al. The efficacy of infection surveillance and control programs in preventing nosocomial infections in US hospitals. Am J Epidemiol 1985;121:182-205.

8. Rosenthal VD, Maki DG, Mehta Y, et al. International Nosocomial Infection Control Consortiu (INICC) report, data summary of 43 countries for 2007-2012. Deviceassociated module. Am J Infect Control 2014;42:942-56.

9. Snyder GM, Morgan DJ. Potential Uses of a National Healthcare-Associated Infection Reporting System. Infect Control Hosp Epidemiol 2016;37:1109-10.

10. Choi HJ, Adiyani L, Sung J, et al. Five-year decreased incidence of surgical site infections following gastrectomy and prosthetic joint replacement surgery through active surveillance by the Korean Nosocomial Infection Surveillance System. J Hosp Infect 2016;93:339-46.

11. Chatterjee A, Yadav P, Varshney S, et al. Study of hand hygiene compliance in intensive care units at a tertiary care hospital. Paripex 2016;5:77-80.

12. Bischoff P, Geffers C, Gastmeier P. Hygienemaßnahmen auf der Intensivstation [Hygiene measures in the intensive care station. Med Klin Intensivmed Notfmed 2014;109:627-39.

13. Gould DJ, Creedon S, Jeanes A, et al. Impact of observing hand hygiene in practice and research: a methodological reconsideration. J Hosp Infect 2017;95:169-74.

14. Levchenko AI, Boscart VM, Fernie GR. The feasibility of an automated monitoring system to improve nurses' hand hygiene. Int J Med Inform 2011;80:596-603.

15. Møller-Sørensen H, Korshin A, Mogensen T, et al. New technology markedly improves hand-hygiene performance among healthcare workers after restroom visits. J Hosp Infect. 2016;92:337-9.
16. Arntz PR, Hopman J, Nillesen M, et al. Effectiveness of a multimodal hand hygiene improvement strategy in the emergency department. Am J Infect Control 2016;44:1203-7.

17. Guideline for hand hygiene. Denver, CO: AORN, Inc., 2017.

18. Koff MD, Corwin HL, Beach ML, et al. Reduction in ventilator associated pneumonia in a mixed intensive care unit after initiation of a novel hand hygiene program. J Crit Care 2011;26:489-95.

19. Pincock T, Bernstein P, Warthman S, et al. Bundling hand hygiene interventions and measurement to decrease health care-associated infections. Am J Infect Control 2012;40:S18-27.

20. National Health Commission's notification of quality control indicator of anesthesiology and other 6 majors. Beijing: National Health Commission, 2015.

21. Ren N, Wen XM, Fu CC, et al. Development and changing trend in monitoring of healthcare-associated infection in China. Chin J Infect Control 2016;15:642-7.

22. You CQ, Li LZ, Zha ZH, et al. Investigation and analysis of quality control indicators for healthcare-associated infections in 118 hospitals of Guizhou province. Chin J Nosocomial 2016;26:4073-6.

23. Liu SL, Wang XC, Zhao SY. The effect of comprehensive intervention measures on multidrug-resistant organism infection among the ICU patients. J Prevent Med Inform 2016;32:1230-3.

24. Xu DH, Hou TY, Li WG, et al. Awareness of hand hygiene knowledge and compliance status in Chinese hospitals. Chin J Infect Control 2016;15:654-8.

25. Li CH, Liu SD, Li LY, et al. Development situation of healthcare-associated infection management departments in the rational antimicrobial application and management in China. Chin J Infect Control 2016;15:665-70.

26. Deng XH, Zhang L, Liu Z, et al. Cross-sectional survey on surgical site infection in 10 hospitals . Chin J Nosocomial 2010;20:1672-3.

27. Zhang J, Xu Q, Wang WH, et al. Analysis on targeted surveillance of surgical site infections and application of antimicrobial agents. Chin J Nosocomial 2015;25:583-4.

28. Wang J, Chen MY, Huang J. Targeted surveillance of nosocomial infections in general ICU. Chin J Nosocomial 2015;25:2242-3.

29. Xu H, Sun J, Gu AM, et al. Prevention and control of catheter-associated urinary tract infection in China. Chin J Infect Control 2016;15:671-5.

30. Allegranzi B, Nejad SB, Combescure C, et al. Burden of endemic health-care-associated infection in developing 
countries: systematic review and meta-analysis. Lancet 2011;377:228-41.

31. Iwuafor AA, Ogunsola FT, Oladele RO, et al. Incidence, Clinical Outcome and Risk Factors of Intensive Care Unit Infections in the Lagos University Teaching Hospital (LUTH), Lagos, Nigeria. PLoS One 2016;11:e0165242.

32. Yilmaz G, Aydin H, Aydin M, et al. Staff education aimed at reducing ventilator-associated pneumonia. J Med Microbiol 2016;65:1378-84.

33. Taylor JS, Marten CA, Munsell MF, et al. The DISINFECT Initiative: Decreasing the Incidence of Surgical Infections in Gynecologic Oncology. Ann Surg Oncol 2017;24:362-8.

Cite this article as: Liu W, Guo T, Li H, Zhao Y, Zhang K, Hai Y, Jiao Y, Xing H, Xu B, Bai H, Bao H, Zhang S, Ren W, Yang L, Yang H, Tian J, Wang M, Yang Y. Healthcareassociated infection prevention and control management in a tertiary hospital and an overall evaluation. Ann Palliat Med 2020;9(4):1536-1544. doi: 10.21037/apm-20-65
34. Leblebicioglu H, Erben N, Rosenthal VD, et al. International Nosocomial Infection Control Consortium (INICC) national report on device-associated infection rates in 19 cities of Turkey, data summary for 2003-2012. Ann Clin Microbiol Antimicrob 2014;13:51.

35. Guan GQ, Xiang DS. Evaluation Hospital Work Quality Using Rank Sum Ratio. Chin J Hosp 2010;17:350-2.

36. Qu W, Deng GZ, Xu TH. Overall evaluation and analysis of hospital medical quality using rank sum ratio. Chin J Hosp 2013;21:88-90.

37. Shi JP, Gulizhaer A, Liu WL, et al. Overall evaluation and analysis of 10 years medical quality of a hospital using rank sum ratio. Chin J Health Statist 2013;30:416-7. 